\title{
Délibérations du Comité central de la FMH
}

Deutsch erschienen

in Nr. 48/2003.
St. Lors de sa séance du 23 octobre 2003, le Comité central de la FMH s'est notamment penché sur les affaires suivantes:

\section{Réforme des structures / droit professionnel}

1. Office fédéral des réfugiés (ODR): mesures d'allègement budgétaire dans le domaine de I'asile: système de monitorage

Pour des raisons faciles à comprendre, l'état de santé des demandeurs d'asile est souvent plus mauvais que celui de la moyenne des Suisses du même âge. Le CC part de l'idée que les décisions en vigueur de non-entrée en matière ne font pas que toucher des demandeurs d'asile en bonne santé. Il considère dès lors l'appréciation selon laquelle ces personnes n'ont besoin qu'«isolément et temporairement» d'aide (médicale) comme étant trop optimiste.

Le CC relève également que les demandeurs d'asile, au cours de leur séjour dans un centre d'accueil, ne bénéficient pas de l'assurance maladie. L'ODR montre de surcroît une tendance à limiter au maximum l'accès aux traitements médicaux, pour des raisons de politique financière en soi compréhensibles. A leur arrivée, ils devraient être assurés comme des sans-papiers lorsqu'ils s'annoncent aux autorités sociales, bien qu'ils risquent alors l'arrestation.

S'agissant de l'aide médicale d'urgence, le concept relève d'emblée que cette aide n'est semble-t-il apportée que dans les hôpitaux, ceuxci étant priés de prendre si possible les frais en charge, donc de les soigner gratuitement. Le CC estime que l'aide médicale d'urgence devrait également avoir lieu dans les cabinets médicaux: la législation sanitaire de tous les cantons prévoit un service médical d'urgence. A cet égard, les médecins praticiens de service sont investis d'un devoir de traitement d'urgence. Comme c'est le cas aujourd'hui pour les sans-papiers, on peut s'attendre à l'avenir à voir une partie de ces patients traités dans les cabinets médicaux et pas uniquement dans le cadre du service d'urgence. Par ailleurs, il convient d'ajouter qu'il n'existe pratiquement pas d'hôpital public en Suisse qui dispose, dans le cadre d'un budget cantonal ou communal, d'un montant à bien plaire pour des traitements médicaux gratuits. Le concept de l'ODR apparaît dès lors quelque peu léger.
Le CC propose que l'ODR intègre la FMH dans l'organisation du système de monitorage pour les questions de l'assistance médicale d'urgence. Il s'agirait d'assurer (de manière contraignante et non aléatoire) le suivi de factures non couvertes aussi bien en milieu hospitalier qu'au cabinet médical (contrairement au concept jusqu'ici en vigueur). Donnant son aval à ce point de vue, le CC prendra contact à ce sujet avec l'ODR.

\section{Modifications de l'ordonnance sur} la circulation routière, $2^{\mathrm{e}}$ train de mesures

Le CC décide de sa prise de position selon laquelle il se limitera aux questions concernant l'inaptitude à conduire. Il relève notamment que la prise de sang doit s'accompagner d'un examen médical. Il n'est pas rare en effet que seuls cet élément complémentaire permette d'attribuer ou non la présumée diminution de l'aptitude à conduire à la seule consommation d'alcool. A cela s'ajoute qu'il n'existe actuellement pas d'indicateur de laboratoire apte à découvrir un quelconque problème de dépendance par la seule prise de sang.

Le CC ne saurait donner son aval à la modification proposée sur les valeurs-limite des produits engendrant la dépendance, car elle ne tient absolument pas compte du recours à des narcotiques à des fins thérapeutiques, dans le traitement de la douleur en particulier.

\section{Assurances sociales}

\section{Mesures d'urgence en psychiatrie}

Dans une lettre au Comité central, les sociétés de discipline psychiatriques réunies dans l'association faîtière FMPP (Foederatio Medicorum Psychiatricorum et Psychotherapeuticorum) exigent des mesures d'urgence immédiates pour la psychiatrie, en ce qui concerne l'introduction du TARMED dans le domaine AA/AM/AI. Concrètement, elle demande une augmentation forfaitaire des positions tarifaires en psychiatrie de $15 \%$.

Le CC note que la compétence en cette affaire relève du G7. La lettre de la FMPP est donc transmise à celui-ci. 


\section{Neutralité des coûts et radiologie}

La Société suisse de radiologie (SSR) demande à la FMH, plus précisément au CC, de sortir le domaine de la radiologie du calcul fédéral et cantonal de la valeur du point et de le traiter comme une entité à part, la progression des coûts devant être analysée et suivie de manière particulière.

Le président de la FMH fait référence à la proposition de la SSR à cet égard lors de la séance de la Chambre médicale extraordinaire du 11.10.2003, à laquelle le CC donnera suite sous forme de base de négociations. Le G7 est convié à négocier pour la radiologie des mesures appropriées dans le domaine de la LAMal, analogues à celles prises dans le domaine de l'AA avec santésuisse.

\section{Table ronde sous la direction du Conseil fédéral, proposition de la FMPP}

Les sociétés de psychiatrie, sous la houlette de la FMPP, ont demandé au CC de la FMH d'organiser prochainement une table ronde sous la direction du Conseil fédéral pour discuter de la situation délétère générée par les négociations TARMED après le RE2.

Le Comité central est convaincu que la situation politique actuelle ne permet pas d'organiser une telle table ronde. Il recommande à la FMPP d'abandonner cette intention. 